(for example, $\quad 3 C 298: z=1 \cdot 436, \quad S_{178}=44 \times 10^{-26}$ $\left.\mathrm{W} \mathrm{m} \mathrm{m}^{-2}(\mathrm{c} / \mathrm{s})^{-1}\right)$.

M. S. LONGaIR

Mullard Radio Astronomy Observatory,

Cavendish Laboratory,

University of Cambridge.

'Hoyle, F., and Burbidge, G. R., Nature, 210, 1346 (1966).

${ }^{2}$ Burbidge, E. M., Paper presented at Erevan Conference on "Instability Phenomena in Galaxies", May 1966

${ }^{3}$ Lynds, C. R., Paper presented at Erevan Conference on "Instability Phenomena in Galaxies", May 1966.

‘ Ryle, M., and Clarke, R. W., Mon. Not. Roy. Astro. Soc., 122, 349 (1961).

${ }^{5}$ Gower, J. F. R., Mon. Not. Roy. Astro. Soc., 133, 151 (1966).

- Penzias, A. A., and Wilson, R. W., Astrophys, J., 142, 419 (1965).

' Roll, P. G., and Wikinson, D. T., Phys. Rev. Lett., 16, 405 (1966).

Field, G. B., and Hitchcock, J. L., Phys. Rev. Lett., 16, 817 (1966).

${ }^{9}$ Howell, T. F., and Shakeshaft, J. R., Nature, 210, 1318 (1966).

${ }^{10}$ Hoyle, F., and Tayler, R. J., Nature, 203, 1108 (1964).

"Hawking, S. W., and Tayler, R. J., Nature, 209, 1339 (1966).

${ }^{12}$ Holden, D. J., Mon. Not. Roy. Astro. Soc., 133, 225 (1966).

${ }^{13}$ Hughes, R. G., and Longair, M. S., Mon. Not. Roy. Astro. Soc. (in the press).

"Faulkner, J., Gunn, J. E., and Peterson, B. A., Nature, 211, 502 (1966).

${ }^{15}$ Ryle, M., Elsmore, B., and Neville, A. C., Nature, 205, 1259 (1965).

18 Véron, P., Astrophys. J., 141, 1284 (1965).

${ }^{17}$ Hazard, C., Mackey, M. B., and Nicholson, W., Nature, 202, 227 (1964).

" Davidson, W., and Davies, M., Mon. Not. Roy. Astro. Soc., 127, 241 (1964).

${ }^{19}$ Longair, M. S., Mon. Nol. Roy. Astro. Soc. (in the press). "V Véron, P., Miami Conference on the "Observational Aspects of Cosmology",

\section{An Apparent Increase in Galactic Radio Noise during a Total Solar Eclipse}

During the total eclipse of 1965, May 30, observations were made on the island of Manuae (lat., $19^{\circ} 15^{\prime} 43^{\prime \prime} \mathrm{S}$.; long., $158^{\circ} 57^{\prime} 43^{\prime \prime} \mathrm{W}$.) of changes in the $D$ layer absorption of radio signals, and also of fluctuations in the galactic noise level at $18.2 \mathrm{Mc} / \mathrm{s}$. The latter observations in particular produced an unexpected effect which is reported here.

The apparatus used in measuring the noise consisted of two half-wave dipoles in phase, with reflectors, the main lobe being directed at R.A. Ih $20 \mathrm{~m}$, Dec. $16^{\circ} 21^{\prime}$ S., that is $8^{\circ}$ from the zenith in a direction W. $20^{\circ} \mathrm{S}$. This aerial fed a low noise receiver of $10 \mathrm{kc} / \mathrm{s}$ bandwidth, and the audio-output was amplified, rectified and used to drive a pen recorder. The system was calibrated with a 5722 noise diode.

Observations were made between $18 \mathrm{~h} 45 \mathrm{~m}$ and $2 \mathrm{lh} 00 \mathrm{~m}$ U.T. on every day between May 27 and June 2. Time markers were received from station $W W V H$ and placed manually on the chart.

The unexpected feature consisted of two large bursts of noise, each of 13 sec duration, the leading edges of which coincided to 3 sec with the times of optical second and third contacts. The mean power level in each burst was about 2.5 times the mean noise power during the eclipse. No such similar bursts occurred at any other time while observations were being made. Because all expeditions on the Island had collaborated in the detection, identification and elimination of any sources of local radio interference, we are satisfied that the noise originated from a source outside the island.

Reduction of the data on this and other observations is not yet complete.

S. G. KINGAN
R. MaEva
T. Simpson
T. UtTANGA

Cook Islands Astronomical Society,

Avarua,

Rarotonga,

Cook Islands.

\section{PLANETARY SCIENCE}

\section{A Method for Translunar Radio Communication}

THE increase of human activity on the lunar surface that can be expected to follow the first manned landings will present a special problem in translunar communications. The lack of an ionosphere about the Moon, the lack of any appreciable atmosphere on the lunar surface, and the nearness of the lunar horizon, require solutions to this problem that may be somewhat different from those most easily available on the Earth's surface. Extended radio coverage about the lithosphere, such as occurs on Earth because of the atmospheric density gradient and temperature inversions, will not be possible because of the vanishingly low density of any gaseous envelope that may exist around the Moon.

Consideration of the special properties which are believed to be likely for the lunar lithosphere ${ }^{1,2}$ indicates a possible solution: that the sub-lunar surface materials can be used as a propagation medium for translunar communication.

The immediate surface layers have an extremely low thermal conductivity and a very low content of liquid water, as shown by microwave thermal observations. Thus there is some evidence that the outer layers of the Moon contain considerable quantities of material similar to earthly pumice, in that this material has been expanded by gas bubbles during some stage of formation, and/or was formed from meteoric splash dust and thermal cycle spallation products, re-welded on succeeding surfaces by direct solar action.

The depth to which these conditions exist is of course at the moment a matter of pure speculation. However, if they persist for an appreciable depth, then a considerable volume of the outer part of the Moon is likely to be trans. parent to radio waves in the range from the lowest frequencies that can be radiated by practical antennae up to frequencies in the tens of megacycles per second. The depth of this transpareney is probably a function of radio frequency, because microwave thermal observations ${ }^{3}$ show some transparency even for microwaves in a thin layer of the outer surface.

Antennae placed on the surface of the Moon should give coupling to sub-surface waves with which astronauts can communicate beyond the lunar horizon. Trailing wire antennae can be simple and inexpensive as compared with arrays of microwave dish antennae, which have the additional disadvantage of being confined to line-of-sight use.

The advantages of sub-lunar low frequency communications seem obvious. Very compact receivers of low mass and transmitters of great reliability are available from simple designs using solid-state active elements and microcircuitry type construction. The principal problem is the primary power. Storage cells and solar cells in combination have already shown their adaptability in the lunar surface environment, in connexion with the Surveyor soft landing and picture transmission.

The remaining problem is that of determining the true nature and extent of the outer portion of the Moon in respect to its radio transmission properties. If the outer layers are sufficiently transparent to a suitable radio frequency, the internal reflexion from the lunar surface and the ray curvature resulting from increased density as a function of depth should ensure a strong wave component along and just below the surface, with sufficient evanescent wave penetration through the surface to ensure antenna coupling.

The foregoing possibilities make it very desirable to obtain measurements of lunar sub-surface properties in relation to radio transparency and the depth of the transparent layers, before the arrival of manned landing parties. This information should be obtainable by means 